\title{
A GPU-based Architecture for Real-Time Data Assessment at Synchrotron Experiments
}

\section{Suren Chilingaryan ${ }^{1}$, Alessandro Mirone ${ }^{2}$, Andrew Hammersley ${ }^{2}$, Claudio Ferrero ${ }^{2}$, Lukas Helfen ${ }^{3}$, Andreas Kopmann ${ }^{1}$, Tomy dos Santos Rolo ${ }^{3}$}

1 Institute for Data Processing and Electronics, Karlsruhe Institute of Technology, Karlsruhe, Germany

${ }^{2}$ European Synchrotron Radiation Facility, Grenoble, France

${ }^{3}$ Institute for Synchrotron Radiation, Karlsruhe Institute of Technology, Karlsruhe, Germany

\section{Fast Tomography at Synchrotron Light Sources}

$\mathrm{X}$-ray imaging permits spatially resolved visualization of $2 \mathrm{D}$ and 3D structures in materials and organisms which is crucial for understanding their properties. Furthermore, it allows one to recognize defects in devices from the macro- down to the nanoscale. Providing millions of pixels, each with a digitization depth of 12 bits or more, and several thousand frames per second, seconds. We have optimized the reconstruction software employed at the micro-tomography beamline at KIT and ESRF to use the computational power of modern graphic cards and reduced reconstruction time from hours down to few minutes.

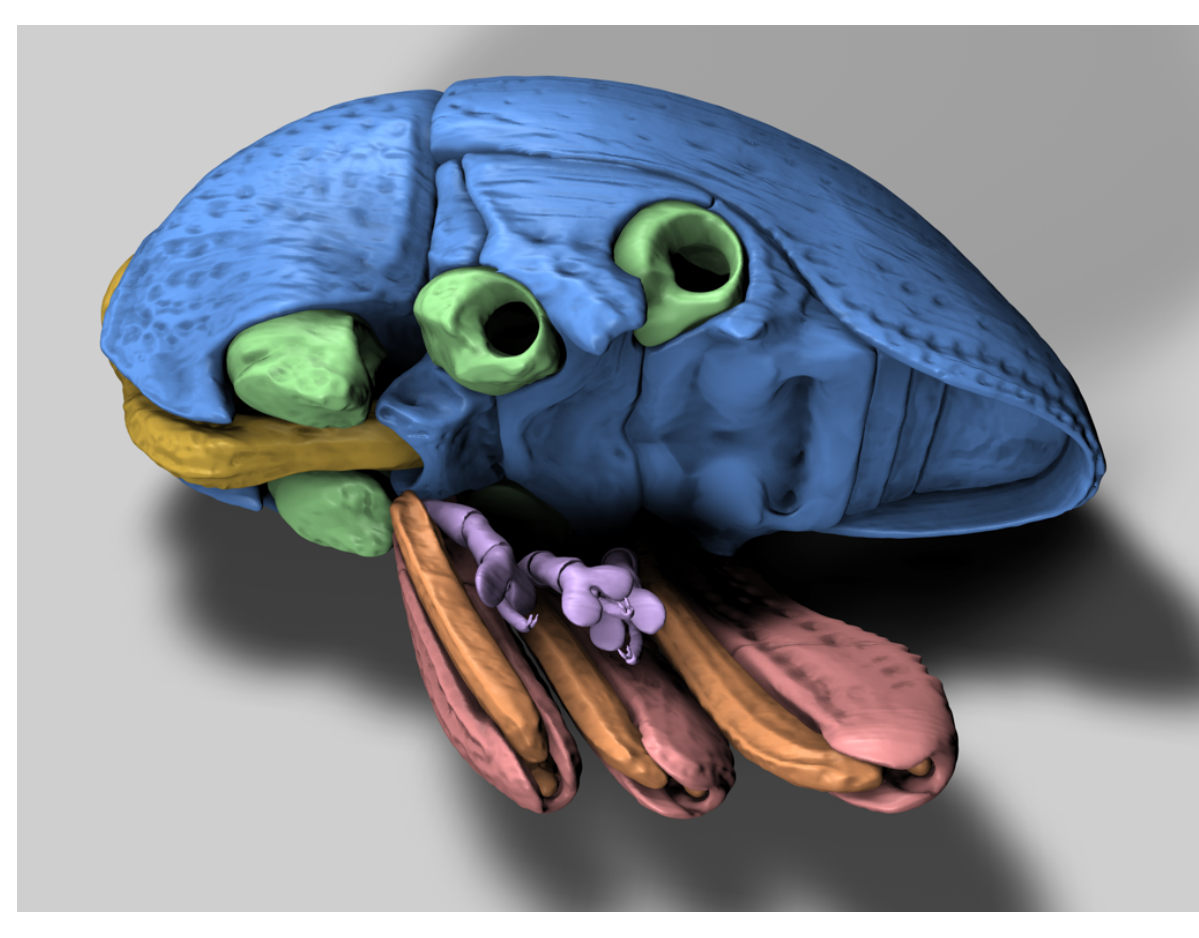

Example for 3D X-Ray imaging. The functional groups of a flightless weev

Imaging at Synchrotron Light Sources

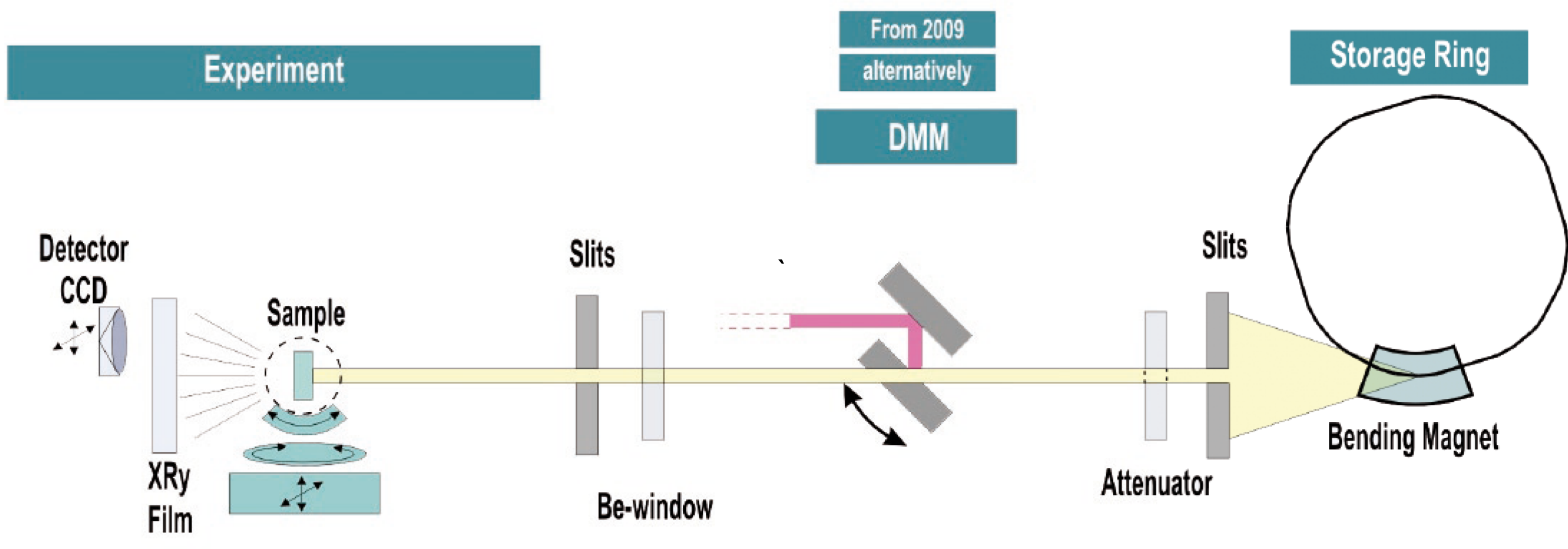

The sample evenly rotating in the front of a pixel detector is penetrated by $X$ rays produced in the synchrotron

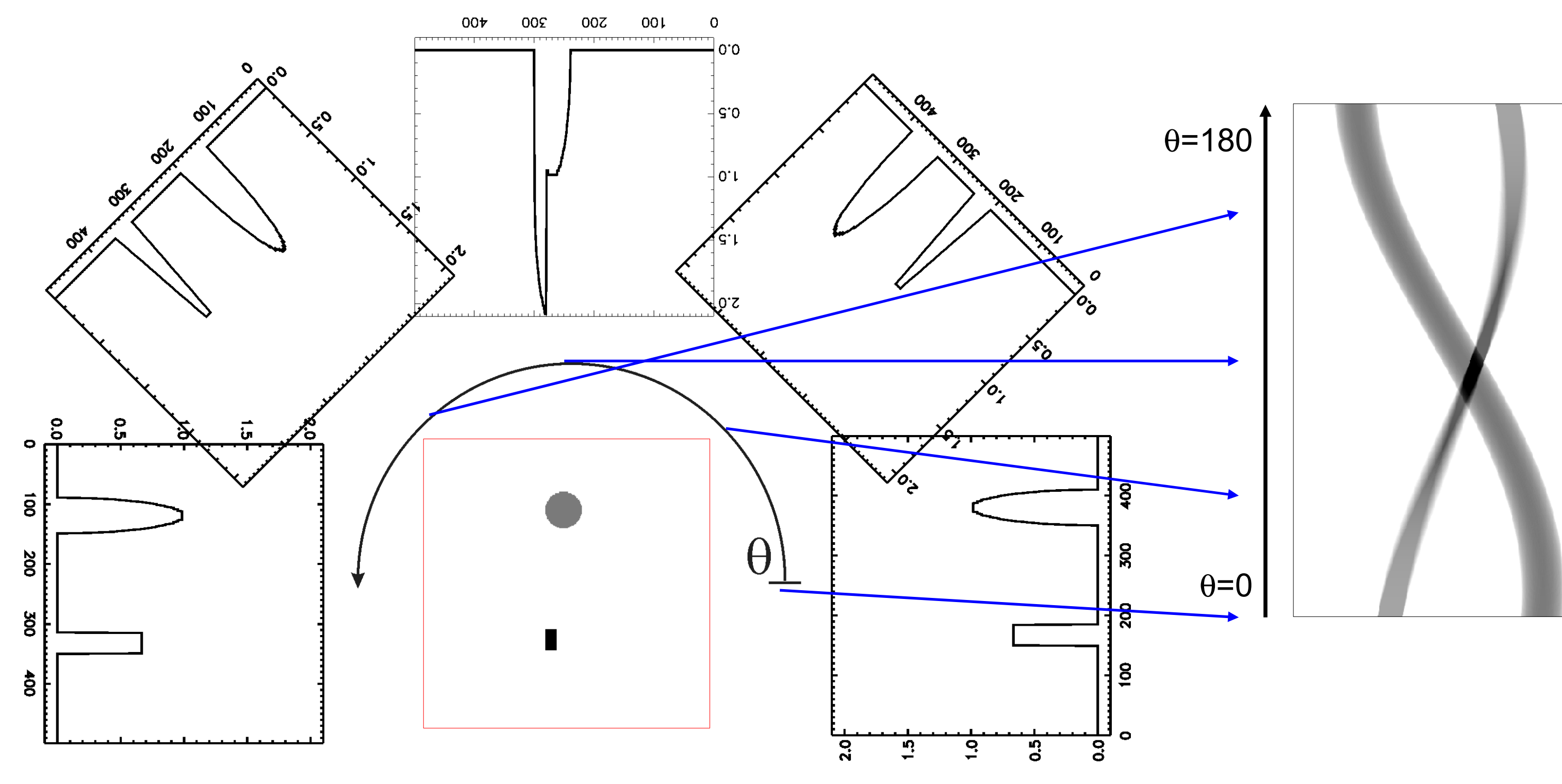

The pixel detector registers series of parallel $2 D$ projections of the sample density at different angles.

3D Image Reconstruction
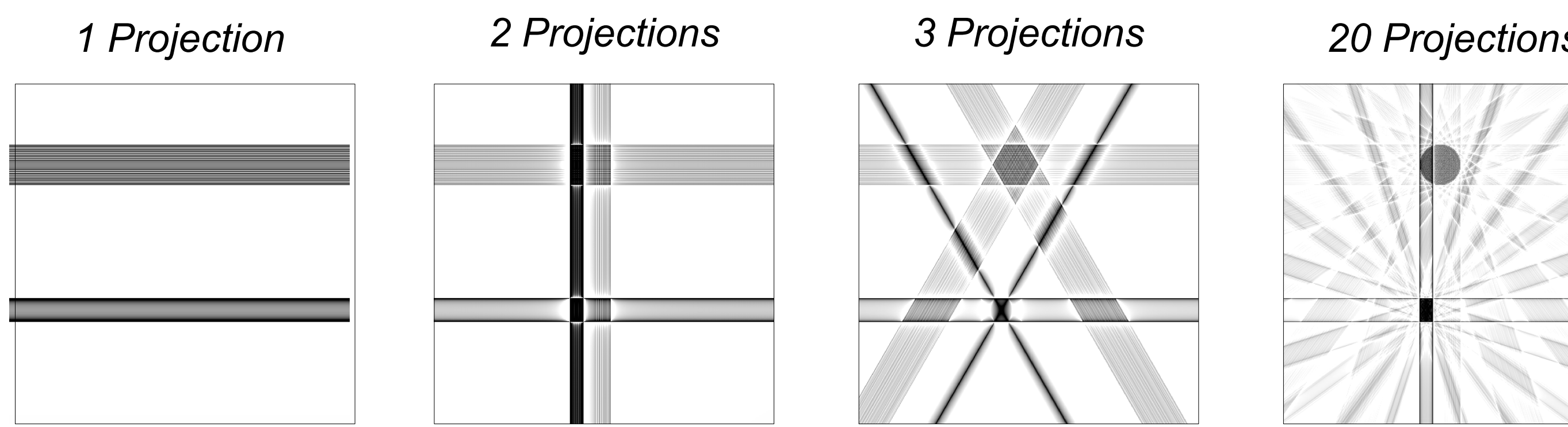

1000 Projections

Filtered back-projection is used to reconstruct $3 D$ images from a manifold of $2 D$ projections. The projection values are smeared back over $2 D$ cross sections and integrated over all projection angles. To reduce blurring effect the projections are filtered in the Fourier space before being back projected.

\section{Example of Typical Setup}

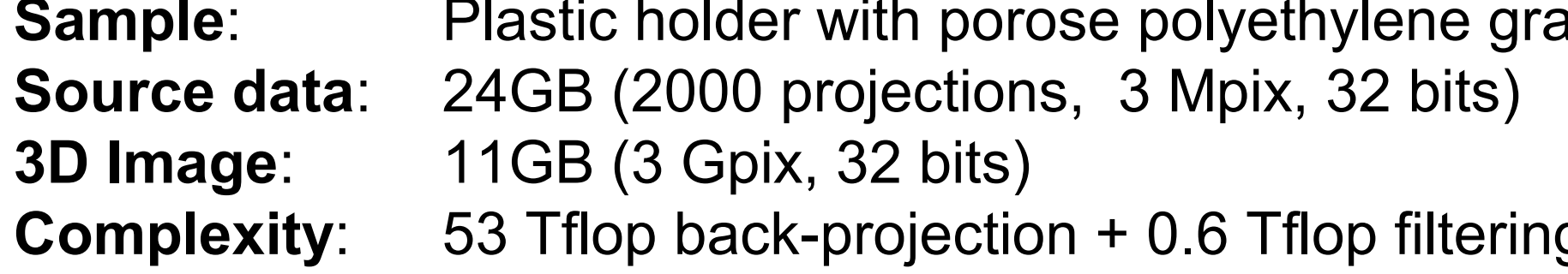

Complexity: $\quad 53$ Tflop back-projection + 0.6 Tflop filtering

Goal: Reconstruct 3D image in 1 minute

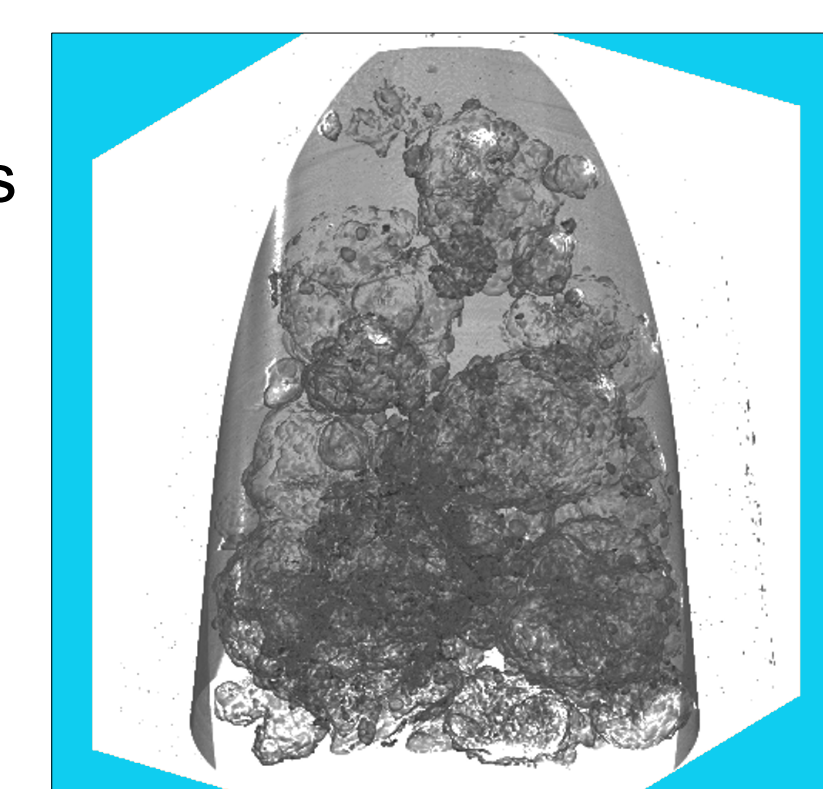

Software Optimizations

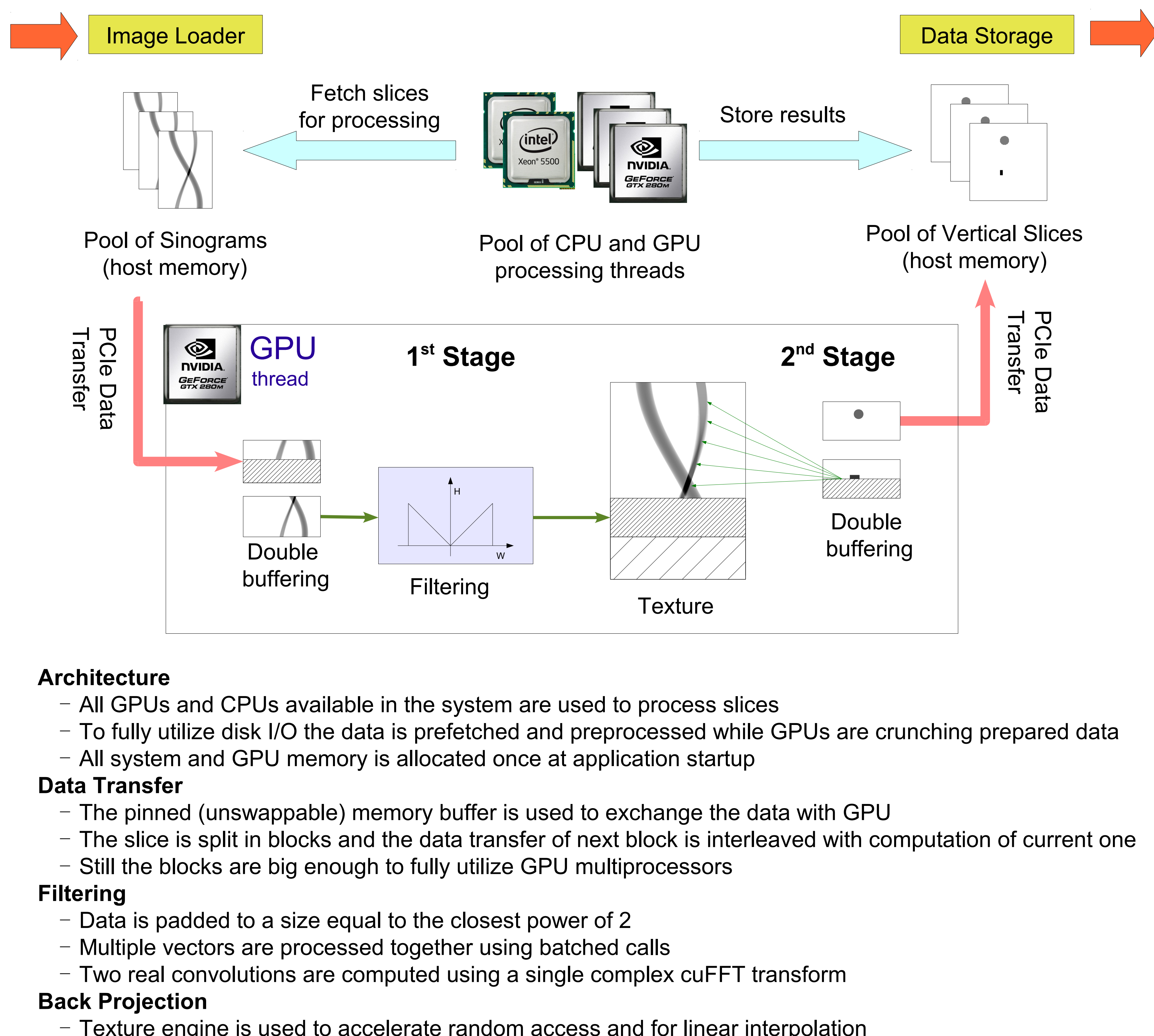

Results

\begin{tabular}{|c|c|c|c|}
\hline & Xeon Server & GPU Desktop & GPU Server \\
\hline $\begin{array}{l}\text { Type of } \\
\text { Computation }\end{array}$ & $\begin{array}{l}\text { CPU / Xeon E5472 } \\
8 \text { core, } 3 \mathrm{GHz}\end{array}$ & $\begin{array}{l}\text { GeForce GTX } 280 \\
1 \text { core }\end{array}$ & $\begin{array}{l}2 \times \text { GTX295 + } 2 \times \text { GTX480 } \\
6 \text { cores }\end{array}$ \\
\hline CPU & $2 \times$ Xeon E5472 & Core2 E6300 & 2 x Xeon E5540 \\
\hline Memory & 16GB DDR3 & 4GB DDR2 & 96GB DDR3 \\
\hline HDD & WDC5000AACS & WDC5000AACS & 2 x Intel X25-E / Raid-0 \\
\hline Price & $5500 \$(2000 \$ C P U)$ & $1000 \$(400 \$$ GPU) & $9000 \$(2000 \$$ GPU, $800 \$$ SSD $)$ \\
\hline Software & \multicolumn{3}{|c|}{ OpenSuSe 11.2, CUDA 3.0, Intel MKL 10.2.1, gcc4.4 -O3 -march=nocona -mfpmath=sse } \\
\hline
\end{tabular}

Scalability
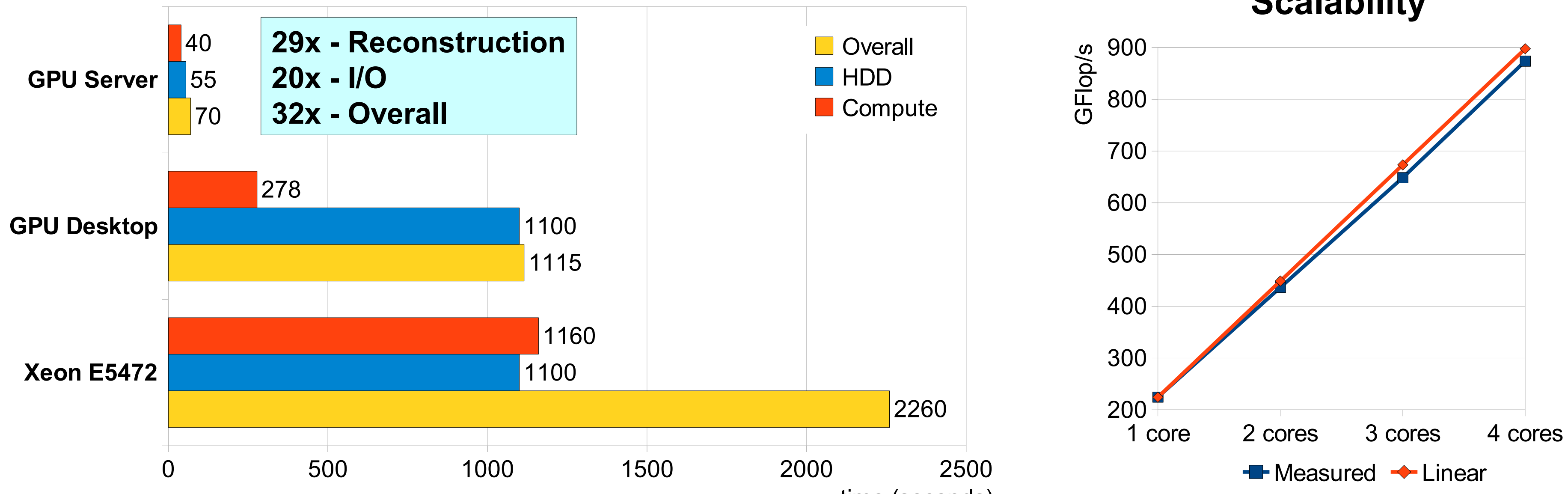

Evaluation of Computation Platforms

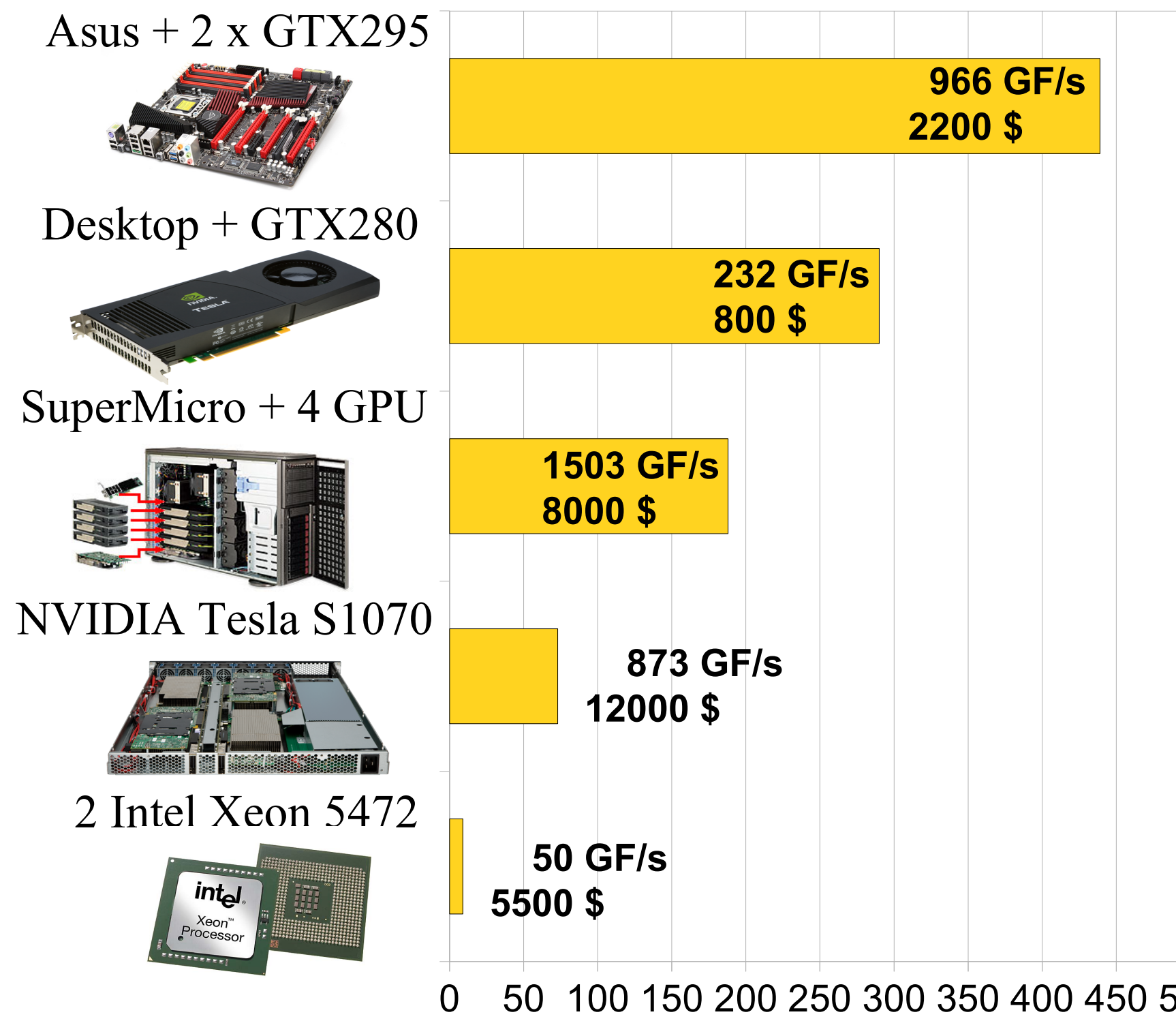

Asus Rampage III Extreme (2200\$ $=$ Core i7 $+2 \times$ GTX295 + 6 GB Chipset: $\times 58,36$ PCle 2.0 lanes; 6 DDR3 slots (48 GB max) Max Peak Performance (NVidia): 7.15 Tflops / 595 GFlops

Standard Desktop $(800 \$=C 0 r e 2+G T \times 285+2 G B$

SuperMicro 7046GT-TRF ( $-8000 \mathrm{~s}=2$ Xeon $+4 \mathrm{GPU}+96 \mathrm{~GB})$ Chipset: Dual Intel 5520, 72 PCle 2.0 lanes, 12 DDR3 slots (192GB max)
PCle 2.0 $26: 4$ (full speed), $\times 4$ : 2 (in $\times 16$ slots); PCle 1.0 44 : (in $\times 8$ slot) NVIDIA Tesla S1070 (-8000\$ +4000 S host) System: Requires separate host server
GPU Devices: 4 × Tesla C1060 (960 parallel proce
Peak performance: 4.14 Thlops 1345 Gflops

GPU Memory Size: $16 \mathrm{~GB}$

Dual Xeon 5472 Server $(5500 \mathrm{~s}=16 \mathrm{~GB})$ 